\title{
A decomposition theorem for fuzzy set-valued random variables
}

\author{
Giacomo Aletti*1,2 and Enea G. Bongiorno ${ }^{\dagger 1}$ \\ ${ }^{1}$ Dipartimento di Matematica, Università degli Studi di Milano \\ ${ }^{2}$ ADAMSS CENTRE (ADvanced Applied Mathematical and Statistical Sciences)
}

December 24, 2012

\begin{abstract}
In this paper, a decomposition theorem for a (square integrable) fuzzy random variable FRV is proposed. The paper is mainly divided in two parts. In the first part, for any FRV $X$, we define the Hukuhara set as the family of (deterministic) fuzzy sets $C$ for which the Hukuhara difference $X \ominus_{H} C$ exists almost surely; in particular, we prove that such a family is a closed (with respect to different well known metrics) convex subset of the family of all fuzzy sets. In the second part, we prove that any square integrable FRV can be decomposed, up to a random translation, as the sum of a FRV $Y$ and an element $C^{\prime}$ chosen uniquely (thanks to a minimization argument) in the Hukuhara set. This decomposition allows us to characterize fuzzy random translations; in particular, a FRV is a fuzzy random translation if and only if its Aumann expectation equals $C^{\prime}$ (given by the above decomposition) up to a deterministic translation. Examples and open problems are also presented.
\end{abstract}

Keywords: Fuzzy random variable; Hukuhara difference; decomposition theorem; randomness defuzzification;

Revised Personal Version of the text of the final journal article:

G. Aletti, E. G. Bongiorno. A decomposition theorem for fuzzy set-valued random variables. Fuzzy Sets and Systems. (doi: 10.1016/j.fss.2012.11.005).

http://dx.doi.org/10.1016/j.fss.2012.11.005

It reflects changes made in the peer review process.

\section{Introduction}

It is widely known (e.g. [6, Theorem 6.1.7]) that a Gaussian fuzzy random variable (FRV) may be decomposed as

$$
X=\mathbb{E} X \oplus \mathbb{I}_{\xi},
$$

where $\mathbb{E} X$ is the expectation of $X$ in the Aumann sense, $\xi$ is a Gaussian random element in $\mathbb{R}^{d}$ with $\mathbb{E} \xi=0$ and $\mathbb{I}_{A}: \mathbb{R}^{d} \rightarrow\{0,1\}$ denotes the indicator function of any $A \subseteq \mathbb{R}^{d}$

$$
\mathbb{I}_{A}(x)= \begin{cases}1, & \text { if } x \in A, \\ 0, & \text { otherwise. }\end{cases}
$$

We write $\mathbb{I}_{a}$ instead of $\mathbb{I}_{\{a\}}$ whenever $A=\{a\}$ is a singleton. Roughly speaking, a Gaussian FRV $X$ is just a deterministic fuzzy set (its expected value $\mathbb{E} X$ ) up to a Gaussian translation $\xi$ which carries out all the randomness of $X$. In this view, Equation (1) entails a randomness defuzzification for the Gaussian FRV $X$ according to which the underlying probability structure can be defined just only on $\mathbb{R}^{d}$ and no

\footnotetext{
* giacomo.aletti@unimi.it
}

†enea.bongiorno@unimi.it 
longer on $\mathbb{F}$, the space of normal fuzzy sets with compact convex level sets. Even the defuzzification is complete both in "shape" and in randomness when one tries to define a fuzzy Brownian motion: if $X$ is a fuzzy Gaussian process with null expectation (in some sense), then $X=\mathbb{I}_{b_{t}}$ with $b_{t}$ a brownian motion in $\mathbb{R}^{d}$, see [2] for details. Clearly, such randomness defuzzification occurs also in the case of a random translation $\xi$ (not necessarily Gaussian) of a deterministic fuzzy set $\nu$; and Equation (1) still holds with $\mathbb{E} X$ being equal $\nu$ up to a translation. From the modeling point of view, the (partial or complete) defuzzification occurrence results in a simplification of the framework chosen to describe the considered phenomenon, so that $X$ can be decomposed as in (1). In other words, defuzzification may tend to mislead the chosen setting: one needs less than a fuzzy framework.

In this paper, we propose a generalization of Equation (1): any FRV $X \in L^{2}[\mathbb{F}]$ can be decomposed by means of

$$
X(\omega)=C \oplus Y(\omega) \oplus \mathbb{I}_{\mathbf{S t e}(X(\omega))},
$$

for $\mathbb{P}$-almost every $\omega \in \Omega$. In particular, we start to consider a $\mathbb{P}$-almost surely centered FRV $X \in L^{2}[\mathbb{F}]$ and the family $\Theta_{X}$ of all centered deterministic $\eta \in \mathbb{F}$ for which the Hukuhara difference $X \ominus_{H} \eta$ exists almost surely. Then, we prove that $\Theta_{X}$ is not empty, convex and closed in $\mathbb{F}$ with respect to $\rho$, where $\rho$ can be chosen between $d_{2}$ (that corresponds to the $L^{2}$ metric in the space of support functions) and $D_{\theta}$ (the mid-spread metric; see [5, 11]). Further,

$$
C=\underset{\eta \in \Theta_{X}}{\arg \min } \mathbb{E}\left[\rho(X, \eta)^{2}\right]
$$

is unique and there exists a ( $\mathbb{P}$-almost surely centered) FRV $Y$ such that $X(\omega)=C \oplus Y(\omega)$. Whenever $X$ is not centered, the decomposition is given by (2). Hence, $C$ and $Y \oplus \mathbb{I}_{\mathbf{S t e}(X)}$ may be considered as the deterministic part (with respect to $\oplus$ ) and the random part of $X$ respectively.

In view of such a decomposition, we are able to handle all kind of defuzzifications: they occur whenever $Y$ or both $C$ and $Y$ are not fuzzy set-valued. In particular, we have a defuzzification in randomness or a complete defuzzification respectively.

In the first case (i.e. $Y$ is $\mathbb{P}$-almost surely $\mathbb{I}_{0}$ ), $X$ is a random fuzzy translation of $C$. Further, because the Aumann expectation $\mathbb{E} X$ is the (unique) Frèchet expectation with respect to $d_{2}$, i.e.

$$
\mathbb{E} X=\underset{\eta \in \mathbb{F}}{\arg \min } \mathbb{E}\left[d_{2}(X, \eta)^{2}\right]
$$

we obtain immediately that a FRV $X$ is a random translation of $C$ if and only if $\mathbb{E} X$ is equal to $C$ up to a translation. (Clearly a fuzzy random translation may be even characterize by its variance: in fact, let $\operatorname{Var}(X)=\mathbb{E}\left[d_{2}(X, \mathbb{E} X)^{2}\right]$ denote the Frèchet variance of the FRV $X$, then $X$ is a fuzzy random translation if and only if $\operatorname{Var}(X-\operatorname{Ste}(X))=0$.)

The existence and uniqueness of decomposition (2) seem to depend on the chosen metric $\rho$. In fact, problems may arise if different metrics are considered, in this paper, besides $d_{2}$ and $D_{\theta}$, we discuss the case of $\rho$ being the Hausdorff metric $\delta_{H}^{\infty}$.

The paper is organized as following. Section 2 introduces necessary notations and literature results. Section 3 studies properties of the Hukuhara set $\Theta_{X}$ whilst Section 4 presents the decomposition theorem of FRV, provides some examples and open problems.

\section{Preliminaries}

Denote by $\mathbb{K}$ the class of non-empty compact convex subsets of $\mathbb{R}^{d}$, endowed with the Hausdorff metric

$$
\delta_{H}(A, B)=\max \left\{\sup _{a \in A} \inf _{b \in B}\|a-b\|, \sup _{b \in B} \inf _{a \in A}\|a-b\|\right\},
$$

and the operations

$$
A+B=\{a+b: a \in A, b \in B\}, \quad \lambda \cdot A=\lambda A=\{\lambda a: a \in A\} \text { with } \lambda>0 .
$$

For a non-empty closed convex set $A \subset \mathbb{R}^{d}$ the support function $s_{A}: S^{d-1} \rightarrow \mathbb{R}$ is defined by

$$
s_{A}(x)=\sup \{\langle x, a\rangle: a \in A\}, \quad \text { for } x \in S^{d-1},
$$


where $\langle\cdot, \cdot\rangle$ is the scalar product in $\mathbb{R}^{d}$ and $S^{d-1}=\left\{x \in \mathbb{R}^{d}:\|x\|=1\right\}$ is the unit sphere in $\mathbb{R}^{d}$. The Steiner point of $A \in \mathbb{K}$ is defined by

$$
\operatorname{ste}(A)=\frac{1}{v_{d}} \int_{S^{d-1}} x s_{A}(x) \mathrm{d} x
$$

where $x \in S^{d-1}$ varies over the unit vectors of $\mathbb{R}^{d}, \mathrm{~d} x$ is the Lebesgue measure on $S^{d-1}$, and $v_{d}$ is the volume of the unit ball of $\mathbb{R}^{d}$.

Fuzzy Sets. A fuzzy set is a map $\nu: \mathbb{R}^{d} \rightarrow[0,1]$. Let $\mathbb{F}$ denote the family of all fuzzy sets $\nu$, which satisfy the following conditions.

1. $\nu$ is an upper semicontinuous function, i.e. for each $\alpha \in(0,1]$, the cut set or the $\alpha$-level set $\nu_{\alpha}=\left\{x \in \mathbb{R}^{d}: \nu(x) \geq \alpha\right\}$ is a closed subset of $\mathbb{R}^{d}$.

2. $\nu$ is normal; i.e. $\nu_{1}=\left\{x \in \mathbb{R}^{d}: \nu(x)=1\right\} \neq \emptyset$.

3. The support set $\nu_{0}=\overline{\left\{x \in \mathbb{R}^{d}: \nu(x)>0\right\}}$ of $\nu$ is compact; hence every $\nu_{\alpha}$ is compact for $\alpha \in(0,1]$.

4. For any $\alpha \in[0,1], \nu_{\alpha}$ is a convex subset of $\mathbb{R}^{d}$.

For any $\nu \in \mathbb{F}$ define the support function of $\nu$ as follows:

$$
s_{\nu}(x, \alpha)=s_{\nu_{\alpha}}(x),
$$

for $(x, \alpha) \in S^{d-1} \times[0,1]$. Let us endow $\mathbb{F}$ with the operations

$$
(\nu \oplus \eta)_{\alpha}=\nu_{\alpha}+\eta_{\alpha}, \quad(\lambda \nu)_{\alpha}=(\lambda \odot \nu)_{\alpha}=\lambda \cdot \nu_{\alpha}, \quad \text { with } \nu, \eta \in \mathbb{F}, \lambda>0 \text { and } \alpha \in[0,1]
$$

(so that $(\mathbb{F}, \oplus, \odot)$ is a convex cone), and with the metrics

$$
\begin{aligned}
\delta_{H}^{\infty}(\nu, \eta) & =\sup \left\{\alpha \in[0,1]: \delta_{H}\left(\nu_{\alpha}, \eta_{\alpha}\right)\right\} \\
d_{2}(\nu, \eta) & =\left(\int_{0}^{1} \int_{S^{d}-1}\left|s_{\nu}(\alpha, u)-s_{\eta}(\alpha, u)\right|^{2} \mathrm{~d} u \mathrm{~d} \alpha\right)^{\frac{1}{2}}
\end{aligned}
$$

where $\mathrm{d} \alpha$ and $\mathrm{d} u$ denote the Lebesgue measure on $[0,1]$ and the normalized Lebesgue measure on $S^{d-1}$ respectively. It is known that $\left(\mathbb{F}, \delta_{H}^{\infty}\right)$ is a complete metric space while $\left(\mathbb{F}, d_{2}\right)$ is not (cf. [4, Chapter 7]); in the following we even use the mid-spread metric $D_{\theta}$ that we introduce later in this section.

Let $\nu, \eta \in \mathbb{F}$, the Hukuhara difference $\nu \ominus_{H} \eta$ is defined by

$$
\nu \ominus_{H} \eta=\tau \quad \Leftrightarrow \quad \tau \oplus \eta=\nu,
$$

whenever $\tau$ exists (in $\mathbb{F}$ ), it is unique.

The generalized Steiner point of $\nu \in \mathbb{F}$ is defined by

$$
\operatorname{Ste}(\nu)=\int_{[0,1]} \operatorname{ste}\left(\nu_{\alpha}\right) \mathrm{d} \alpha
$$

where $\mathrm{d} \alpha$ is the Lebesgue measure on $[0,1]$. In other words, $\operatorname{Ste}(\nu)$ may be seen as a weighted average of steiner points of the level sets of $\nu$. The following properties are satisfied (cf. [12]).

1. For any $\nu \in \mathbb{F}, \operatorname{Ste}(\nu) \in \nu_{0}$.

2. For any $\nu, \eta \in \mathbb{F}, \mathbf{S t e}(\nu \oplus \eta)=\operatorname{Ste}(\nu)+\operatorname{Ste}(\eta)$.

3. Ste $: \mathbb{F} \rightarrow \mathbb{R}^{d}$ is continuous with respect to $\delta_{H}^{\infty}$ (and hence with respect to $d_{2}$ ) and to the euclidean distance on $\mathbb{R}^{d}$. 
On the support functions of fuzzy sets; Bobylev's characterization of a fuzzy set. The support function for a fuzzy set $\nu \in \mathbb{F}$ can be defined equivalently on the closed unit ball $B(0,1)=$ $\left\{x \in \mathbb{R}^{d}:\|x\| \leq 1\right\} \subset \mathbb{R}^{d}$ instead of the unit sphere $S^{d-1}$ by

$$
\begin{aligned}
s_{\nu}^{*}: \quad B(0,1) & \rightarrow \mathbb{R} \\
x & \mapsto s_{\nu}^{*}(x)=\max \left\{\langle x, y\rangle: y \in \mathbb{R}^{d}, \nu(y) \geq\|x\|\right\} .
\end{aligned}
$$

Note that the maximum is actually attained since it is being applied to a linear function over the compact set $\nu_{\|x\|}$. The first step is to analyze the relationship between both definitions of support function. The following results gather this relationship.

Proposition 1 Let $\nu \in \mathbb{F}$. Let $s_{\nu}$ and $s_{\nu}^{*}$ be defined by Equation (3) and Equation (4) respectively.

- If we define $f: S^{d-1} \times[0,1] \rightarrow \mathbb{R}$ as

$$
f(x, \alpha)= \begin{cases}\frac{1}{\alpha} s_{\nu}^{*}(\alpha x), & \text { if } \alpha \neq 0, \\ \sup _{y \in \nu_{0}}\langle y, x\rangle, & \text { if } \alpha=0,\end{cases}
$$

then $f=s_{\nu}$.

- If we define $f^{*}: B(0,1) \rightarrow \mathbb{R}$ as

$$
f^{*}(x)= \begin{cases}\|x\| s_{\nu}\left(\frac{x}{\|x\|},\|x\|\right), & \text { if } x \neq 0 \\ 0, & \text { if } x=0\end{cases}
$$

then $f^{*}=s_{\nu}^{*}$

Proof. First case. For all $\alpha \in(0,1]$, we have

$$
\begin{aligned}
f(x, \alpha)=\frac{1}{\alpha} s_{\nu}^{*}(\alpha x) & =\frac{1}{\alpha} \max \left\{\langle\alpha x, y\rangle: y \in \mathbb{R}^{d}, \nu(y) \geq\|\alpha x\|=\alpha\right\} \\
& =\max \left\{\langle x, y\rangle: y \in \mathbb{R}^{d}, \nu(y) \geq \alpha\right\}=\sup \left\{\langle x, y\rangle: y \in \nu_{\alpha}\right\}=s_{\nu}(x, \alpha) .
\end{aligned}
$$

Finally, by definition, $f(x, 0)=s_{\nu}(x, 0)$ for each $x \in S^{d-1}$.

Second case. Let $x \in B(0,1) \backslash\{0\}$, thus we have

$$
\begin{aligned}
f^{*}(x) & =\|x\| s_{\nu}\left(\frac{x}{\|x\|},\|x\|\right)=\|x\| \sup \left\{\left\langle\frac{x}{\|x\|}, y\right\rangle: y \in \nu_{\|x\|}\right\} \\
& =\max \left\{\langle x, y\rangle: y \in \nu_{\|x\|}\right\}=\max \left\{\langle x, y\rangle: y \in \mathbb{R}^{d}, \nu(y) \geq\|x\|\right\}=s_{\nu}^{*}(x) .
\end{aligned}
$$

Finally, $f^{*}(0)=0=s_{\nu}^{*}(0)$.

Since $s_{\nu}$ and $s_{\nu}^{*}$ are equivalent ways to identify a fuzzy sets, they can be used interchangeably. In the following we will use mainly $s_{\nu}$. We will use $s_{\nu}^{*}$ only in the first part of the proof of Proposition 5 in combination with the Bobylev's characterization for fuzzy sets (Proposition 2).

Proposition 2 (Bobylev's characterization, $[1,4]$ ) Let $f^{*}: B(0,1) \rightarrow \mathbb{R}$. It is a support function (as defined by Equation (4)) of some fuzzy set $\nu \in \mathbb{F}$ if and only if $f^{*}$ satisfies the following properties

(P-1) $f^{*}$ is upper semicontinuous, i.e.,

$$
f^{*}(x)=\limsup _{y \rightarrow x} f^{*}(y), \quad \forall x \in B(0,1) .
$$

(P-2) $f^{*}$ is positively semihomogeneous, i.e.,

$$
\lambda f^{*}(x) \leq f^{*}(\lambda x), \quad \forall \lambda \in(0,1], \forall x \in B(0,1) .
$$

(P-3) $f^{*}$ is quasiadditive, i.e.,

$$
\|x\| f^{*}\left(\lambda \frac{x}{\|x\|}\right) \leq\left\|x_{1}\right\| f^{*}\left(\lambda \frac{x_{1}}{\left\|x_{1}\right\|}\right)+\left\|x_{2}\right\| f^{*}\left(\lambda \frac{x_{2}}{\left\|x_{2}\right\|}\right),
$$

for every $\lambda \in(0,1]$, and $x, x_{1}, x_{2} \in \mathbb{R}^{d} \backslash\{0\}$, with $x=x_{1}+x_{2}$. 
(P-4) $f^{*}$ is normal, i.e.,

$$
f^{*}(x)+f^{*}(-x) \geq 0, \quad \forall x \in B(0,1) .
$$

(P-5) $f^{*}(\cdot) /\|\cdot\|$ is bounded, i.e.,

$$
\sup \left\{\left|f^{*}(x)\right| /\|x\|: x \in B(0,1) \backslash\{0\}\right\}<+\infty .
$$

$(\boldsymbol{P}-6) f^{*}(0)=0$.

In particular, $\nu \in \mathbb{F}$ is such that,

- for $x \in \mathbb{R}^{d}, \nu(x)=\max \left\{\alpha \in[0,1]:\langle x, y\rangle \leq f^{*}(y), \forall y \in B(0,1),|y|=\alpha\right\}$,

- for $\alpha \in(0,1], \nu_{\alpha}=\left\{x \in \mathbb{R}^{d}:\langle x, y\rangle \leq f^{*}(y), \forall y \in B(0,1),|y|=\alpha\right\}$,

- $\nu_{0}=\bigcup_{\alpha \in(0,1]} \nu_{\alpha}$.

Embeddings and metrics. Let $C\left(S^{d-1}\right)$ denote the Banach space of all continuous functions $v$ on $S^{d-1}$ with respect to the norm $\|v\|_{C}=\sup _{x \in S^{d-1}}|v(x)|$. Let $\overline{\mathbf{C}}:=\bar{C}\left([0,1], C\left(S^{d-1}\right)\right)$ be the set of all functions $f:[0,1] \rightarrow C\left(S^{d-1}\right)$ such that $f$ is bounded, left continuous with respect to $\alpha \in(0,1]$, right continuous at 0 , and $f$ has right limit for any $\alpha \in(0,1)$. Then we have that $\overline{\mathbf{C}}$ is a Banach space with the norm $\|f\|_{\bar{C}}=\sup _{\alpha \in[0,1]}\|f(\alpha)\|_{C}$.

Let $\mathcal{L}:=L^{2}\left[[0,1] \times S^{d-1} ; \mathbb{R}\right]$ be the Hilbert space of square integrable real-valued functions defined on $[0,1] \times S^{d-1}$ and let us denote by $\langle\cdot, \cdot\rangle_{\mathcal{L}}$ and $\|\cdot\|_{\mathcal{L}}$ its usual inner product and norm respectively.

It is known, cf. $[6,9,10]$, that the injection $j$ defined by

$$
\begin{aligned}
j: \mathbb{F} & \rightarrow \overline{\mathbf{C}} \cap \mathcal{L} \\
\nu & \mapsto j(\nu)=s_{\nu},
\end{aligned}
$$

satisfies the following properties:

1. $j\left(\lambda_{1} \nu \oplus \lambda_{2} \eta\right)=\lambda_{1} j(\nu)+\lambda_{2} j(\eta), \nu, \eta \in \mathbb{F}$ and $\lambda_{1}, \lambda_{2} \geq 0$.

2. $j$ is an isometric mapping, i.e. for every $\nu, \eta \in \mathbb{F}$,

$$
\delta_{H}^{\infty}(\nu, \eta)=\|j(\nu)-j(\eta)\|_{\bar{C}}, \quad \text { and } \quad d_{2}(\nu, \eta)=\|j(\nu)-j(\eta)\|_{\mathcal{L}} .
$$

The mid-spread metric $D_{\theta}^{\varphi}$, introduced by [11] and extended in [5], is defined as follow. Let $f \in \mathcal{L}$, it can be decomposed as $f=\operatorname{mid} f+\operatorname{spr} f$ where

$$
\operatorname{mid} f(u, \alpha)=\frac{f(u, \alpha)-f(-u, \alpha)}{2}, \quad \operatorname{spr} f(u, \alpha)=\frac{f(u, \alpha)+f(-u, \alpha)}{2},
$$

with $(u, \alpha) \in S^{d-1} \times[0,1]$. Let $\theta \in(0, \infty)$. For $f, g \in \mathcal{L}$ consider the value

$$
\langle f, g\rangle_{\theta}^{\varphi}=[\operatorname{mid} f, \operatorname{mid} g]^{\varphi}+\theta[\operatorname{spr} f, \operatorname{spr} g]^{\varphi},
$$

with

$$
[f, g]^{\varphi}=\int_{0}^{1} \int_{S d-1} f(u, \alpha) g(u, \alpha) \mathrm{d} u \mathrm{~d} \varphi(\alpha),
$$

where $\varphi$ is a weighting probability measure, absolutely continuous (with respect to the Lebesgue measure $\mathrm{d} \alpha)$ probability measure on $\left([0,1], \mathcal{B}_{[0,1]}\right)$ with positive mass function in $(0,1) .\left(\mathcal{L},\langle\cdot, \cdot\rangle_{\theta}^{\varphi}\right)$ is a separable Hilbert space (whose norm is denoted by $\|\cdot\|_{\theta}^{\varphi}$ ). Then, embedding $j: \mathbb{F} \rightarrow \mathcal{L}$ leads to the following metric definition on $\mathbb{F}$

$$
D_{\theta}^{\varphi}(\nu, \eta)=\left\langle s_{\nu}-s_{\eta}, s_{\nu}-s_{\eta}\right\rangle_{\theta}^{\varphi}=\left(\left\|s_{\nu}-s_{\eta}\right\|_{\theta}^{\varphi}\right)^{2},
$$

for any $\nu, \eta \in \mathbb{F}$. We omit $\varphi$ and we write $\langle\cdot, \cdot\rangle_{\theta},\|\cdot\|_{\theta}$ and $D_{\theta}$ whenever $\varphi$ is the Lebesgue measure on $[0,1]$. Note that, the topology induced by $D_{\theta}$ on $\mathbb{F}$ is equivalent to the one induced by $d_{2}$ :

$$
\begin{array}{ll}
\left(\|f\|_{\mathcal{L}}\right)^{2} \leq\left(\|f\|_{\theta}\right)^{2} \leq \theta\left(\|f\|_{\mathcal{L}}\right)^{2}, & \text { if } \theta>1, \\
\theta\left(\|f\|_{\mathcal{L}}\right)^{2} \leq\left(\|f\|_{\theta}\right)^{2} \leq\left(\|f\|_{\mathcal{L}}\right)^{2}, & \text { if } 0<\theta \leq 1
\end{array}
$$

Note that $\|\cdot\|_{\theta}^{\varphi}$ remains equivalent to $\|\cdot\|_{\mathcal{L}}$ in a lot of likely situations (as example, whenever the Radon-Nikodym derivative satisfies $0<h \leq \frac{\mathrm{d} \varphi}{\mathrm{d} \alpha} \leq H$ for some $h, H \in \mathbb{R}$ ). 
Fuzzy random variables. Let $(\Omega, \mathfrak{F}, \mathbb{P})$ be a complete probability space. A fuzzy set-valued random variable (FRV) is a function $X: \Omega \rightarrow \mathbb{F}$, such that $X_{\alpha}: \omega \mapsto X(\omega)_{\alpha}$ are random compact convex sets for every $\alpha \in(0,1]$ (i.e. $X_{\alpha}$ is a $\mathbb{K}$-valued function measurable w.r.t. $\mathcal{B}_{\mathbb{K}}$, the Borel $\sigma$-algebra on $\mathbb{K}$ generated by the metric $\delta_{H}$ ). It has been proven in [3] that this measurability definition is equivalent to the $\mathcal{B}\left(\mathbb{F}, d_{2}\right)$-measurability, hence to the $\mathcal{B}\left(\mathbb{F}, D_{\theta}\right)$-measurability, and, it is necessary (but not sufficient) for the $\mathcal{B}\left(\mathbb{F}, \delta_{H}^{\infty}\right)$-measurability, where $\mathcal{B}(\mathbb{F}, D)$ is the Borel $\sigma$-algebra defined on $\mathbb{F}$ w.r.t. the metric $D$. As a consequence of continuity of $\operatorname{Ste}(\cdot)$, if $X$ is a $\mathrm{FRV}$, then $\mathbf{S t e}(X)$ is a random element in $\mathbb{R}^{d}$.

A FRV $X$ is integrably bounded and we write $X \in L^{1}[\Omega ; \mathbb{F}]$, if $\mathbb{E}\left[\sup _{x \in X_{0}}\|x\|\right]<+\infty$. The (Aumann) expected value of $X \in L^{1}[\Omega ; \mathbb{F}]$, denoted by $\mathbb{E}[X]$, is a fuzzy set such that, for every $\alpha \in[0,1]$,

$$
(\mathbb{E}[X])_{\alpha}=\int_{\Omega} X_{\alpha} \mathrm{d} \mathbb{P}=\left\{\mathbb{E}(f): f \in L^{1}\left[\Omega ; \mathbb{R}^{d}\right], f \in X_{\alpha} \mathbb{P} \text { - a.e. }\right\} .
$$

It should be pointed out that, whenever $\mathbb{E}\left[\left(\sup _{x \in X_{0}}\|x\|\right)^{2}\right]<+\infty$ (we write $X \in L^{2}[\Omega ; \mathbb{F}]$ ), the expected value in the Aumann's sense is even the Frèchet expectation with respect to $d_{2}$ (e.g. [8]), i.e.

$$
\mathbb{E} X=\underset{\eta \in \mathbb{F}}{\arg \min } \mathbb{E}\left[d_{2}(X, \eta)^{2}\right]
$$

In view of above measurability consideration and from embedding (5) it follows that every FRV $X$ can be regarded as a random element in $\mathcal{L}$, where $s_{X}(\cdot, \cdot)(\omega)=s_{X(\omega)}(\cdot, \cdot)$. Moreover, if $X \in L^{1}[\Omega ; \mathbb{F}]$, for any $(x, \alpha) \in \mathbb{R}^{d} \times[0,1], s_{X(\cdot)}(x, \alpha) \in L^{1}[\Omega ; \mathbb{R}]$ and

$$
\mathbb{E}\left[s_{X}(x, \alpha)\right]=s_{\mathbb{E} X}(x, \alpha) .
$$

Finally, let $L^{2}[\Omega ; \mathcal{L}]:=\left\{f: \Omega \rightarrow \mathcal{L}\right.$ s.t. $\left.\left(\mathbb{E}\left[\|f\|_{\mathcal{L}}^{2}\right]\right)^{1 / 2}<+\infty\right\}$. It is an Hilbert space with inner product $\mathbb{E}\left[\langle f, g\rangle_{\mathcal{L}}\right]$. Then, the map

$$
\begin{aligned}
J: \quad L^{2}[\Omega ; \mathbb{F}] & \rightarrow L^{2}[\Omega ; \mathcal{L}] \\
X & \mapsto J(X)=j(X(\cdot))=s_{X(\cdot)}
\end{aligned}
$$

is well-defined and induces an isometry on $L^{2}[\Omega ; \mathcal{L}]$; in particular, for every $X, Y \in L^{2}[\Omega ; \mathbb{F}]$,

$$
\left(\Delta_{2}(X, Y)\right)^{2}:=\mathbb{E}\left[d_{2}(X, Y)^{2}\right]=\mathbb{E}\left[\langle J(X)-J(Y), J(X)-J(Y)\rangle_{\mathcal{L}}\right]
$$

Clearly, $L^{2}[\Omega ; \mathbb{F}]$ is, by means of $J$, even isometric to (the Hilbert space) $\left(L^{2}[\Omega ; \mathcal{L}], \mathbb{E}\left[\langle\cdot, \cdot\rangle_{\theta}\right]\right)$, with

$$
\left(\Delta_{2, \theta}(X, Y)\right)^{2}:=\mathbb{E}\left[D_{\theta}(X, Y)^{2}\right]=\mathbb{E}\left[\langle J(X)-J(Y), J(X)-J(Y)\rangle_{\theta}\right] .
$$

\section{Hukuhara set}

In this section we define the Hukuhara set associated to a FRV $X$, namely $\Theta_{X}$. We provide some properties of $\Theta_{X}$ most of which turn out to be useful in the next section where a decomposition theorem for fuzzy random variables is set.

Let $\nu$ be in $\mathbb{F}$ such that $\operatorname{Ste}(\nu)=0$ and consider

$$
\begin{aligned}
\theta_{\nu} & =\left\{\eta \in \mathbb{F}: \operatorname{Ste}(\eta)=0 \text { and } \exists\left(\nu \ominus_{H} \eta\right)\right\} \\
& =\{\eta \in \mathbb{F}: \operatorname{Ste}(\eta)=0 \text { and } \exists \tau \in \mathbb{F} \text { s.t. } \eta \oplus \tau=\nu\} ;
\end{aligned}
$$

i.e. the family of those centered convex compact fuzzy sets $\eta$ for which the Hukuhara difference $\nu \ominus_{H} \eta$ does exist. Note that $\theta_{\nu}$ is not empty, since $\mathbb{I}_{0}, \nu \in \theta_{\nu}$ and $\{\lambda \odot \nu\}_{\lambda \in[0,1]} \subseteq \theta_{\nu}$. Clearly, if $\eta \in \theta_{\nu}$ and $\tau$ is the Hukuhara difference between $\nu$ and $\eta$, then $\tau \in \theta_{\nu}$.

Proposition $3 \quad \theta_{\nu}$ is a closed subset in $\left(\mathbb{F}, \delta_{H}^{\infty}\right)$.

Proof. Let $\left\{\eta_{n}\right\} \subset \theta_{\nu}$ be a convergent sequence with limit $\eta \in \mathbb{F}$ with respect to $\delta_{H}^{\infty}$, we have to prove that $\eta \in \theta_{\nu}$. Equivalently, we have to prove that there exists $\tau \in \mathbb{F}$ such that $\eta \oplus \tau=\nu$. For each 
$n=1,2, \ldots$ there exist $\tau_{n} \in \mathbb{F}$ such that $\eta_{n} \oplus \tau_{n}=\nu$. Thus, the idea is to prove that $\left\{\tau_{n}\right\}_{n=1}^{\infty}$ converges, w.r.t. $\delta_{H}^{\infty}$, to some $\tau \in \mathbb{F}$ such that $\eta \oplus \tau=\nu$. Note that

$$
\begin{aligned}
\delta_{H}^{\infty}\left(\tau_{m}, \tau_{n}\right) & =\left\|s_{\tau_{m}}-s_{\tau_{n}}\right\|_{\bar{C}} \\
& =\left\|\left(s_{\tau_{m}}+s_{\eta_{m}}\right)-\left(s_{\tau_{n}}+s_{\eta_{n}}\right)+s_{\eta_{n}}-s_{\eta_{m}}\right\|_{\bar{C}} \\
& =\left\|s_{\nu}-s_{\nu}+s_{\eta_{n}}-s_{\eta_{m}}\right\|_{\bar{C}} \\
& =\left\|s_{\eta_{n}}-s_{\eta_{m}}\right\|_{\bar{C}}=\delta_{H}^{\infty}\left(\eta_{n}, \eta_{m}\right) \rightarrow 0, \quad \text { for } n, m \rightarrow \infty
\end{aligned}
$$

where we use the isometry $\tau \mapsto s_{\tau}$ (first and last equalities) and the fact that $\eta_{n}, \eta_{m}$ belong to $\theta_{\nu}$ (third equality). Thus $\left\{\tau_{n}\right\}_{n \in \mathbb{N}}$ is a Cauchy sequence in $\left(\mathbb{F}, \delta_{H}^{\infty}\right)$ that is a complete metric space (e.g. [6, Theorem 5.1.6]), and hence there exists $\tau$ in $\mathbb{F}$ such that $\tau_{n} \rightarrow \tau$. As a consequence, $\eta_{n} \oplus \tau_{n} \rightarrow \eta \oplus \tau$ for $n \rightarrow \infty$ combined with

$$
0=\delta_{H}^{\infty}\left(\eta_{n} \oplus \tau_{n}, \nu\right)
$$

guarantees that $\eta \oplus \tau=\nu$ and hence $\eta \in \theta_{\nu}$; that is the thesis.

In what follows we need the next lemma according to which a fuzzy set can be defined starting from its $\alpha$-cuts.

Lemma 4 (See [4, Proposition 6.1.7, p.39]) If $\left\{C_{\alpha}\right\}_{\alpha \in[0,1]}$ satisfies

(a) $C_{\alpha}$ is a non empty, compact, convex subset of $\mathbb{R}^{d}$, for every $\alpha \in[0,1]$;

(b) $C_{\beta} \subseteq C_{\alpha}$ for $0 \leq \alpha \leq \beta \leq 1$;

(c) $C_{\alpha}=\bigcap_{i=1}^{\infty} C_{\alpha_{i}}$ for all sequences $\left\{\alpha_{i}\right\}_{i \in \mathbb{R}}$ in $[0,1]$ converging from below to $\alpha$, i.e. $\alpha_{i} \uparrow \alpha$ in $[0,1]$; then the function

$$
\nu(x)= \begin{cases}0, & \text { if } x \notin C_{0} \\ \sup \left\{\alpha \in[0,1]: x \in C_{\alpha}\right\}, & \text { if } x \in C_{0}\end{cases}
$$

is an element of $\mathbb{F}$ with $\nu_{\alpha}=C_{\alpha}$ for any $\alpha \in(0,1]$ and

$$
\nu_{0}=\overline{\bigcup_{\alpha \in(0,1]} C_{\alpha}} \subseteq C_{0}
$$

Let $X$ be a FRV. For the sake of simplicity and without loss of generality, let us suppose that $\operatorname{Ste}(X)=0$; otherwise one can always consider its associated centered FRV $\tilde{X}=X-\mathbb{I}_{\text {Ste }(X)}$. The next theorem defines the Hukuhara set $\Theta_{X}$ associated to $X$, and provides some properties of $\Theta_{X}$.

Proposition 5 Let $X$ be a FRV with $\mathbf{S t e}(X)=0$. If $\eta \in \mathbb{F}$, then $E=\left\{\eta \in \theta_{X}\right\}:=\left\{\omega \in \Omega: \eta \in \theta_{X}(\omega)\right\}$ is measurable in $(\Omega, \mathfrak{F})$. Moreover, if $\Theta_{X}=\left\{\eta \in \mathbb{F}: \mathbb{P}\left(\eta \in \theta_{X}\right)=1\right\}$, then the following statements hold.

(i) $\Theta_{X}$ is non-empty: $\mathbb{I}_{0} \in \Theta_{X}$.

(ii) $\eta \in \Theta_{X}$ if and only if there exist a FRV $Y$ such that $\eta \oplus Y=X, \mathbb{P}-$ a.s. If $X \in L^{2}[\Omega ; \mathbb{F}]$, then $Y$ is in $L^{2}[\Omega ; \mathbb{F}]$ too.

(iii) $\Theta_{X}$ is a convex subset in $(\mathbb{F}, \oplus)$. As a consequence, if $\eta \in \Theta_{X}$, then $\{\lambda \eta\}_{\lambda \in[0,1]} \subseteq \Theta_{X}$ with $0 \cdot \eta=\mathbb{I}_{0} \in \Theta_{X}$.

(iv) $\Theta_{X}$ is a closed subset of $\left(\mathbb{F}, \delta_{H}^{\infty}\right)$.

(v) $\Theta_{X}$ is a closed subset of $\left(\mathbb{F}, d_{2}\right)$.

(vi) $\Theta_{X}$ is a closed subset of $\left(\mathbb{F}, D_{\theta}\right)$ and, more in general, of $\left(\mathbb{F}, D_{\theta}^{\varphi}\right)$.

Proof. By definition of $\theta_{X(\omega)}$, we have

$$
\begin{aligned}
E & =\left\{\omega \in \Omega: \operatorname{Ste}(\eta)=0 \text { and } \exists\left(X(\omega) \ominus_{H} \eta\right)\right\} \\
& =\{\omega \in \Omega: \operatorname{Ste}(\eta)=0\} \cap\left\{\omega \in \Omega: \exists Y_{\omega} \in \mathbb{F}, \text { s.t. } s_{\eta}+s_{Y_{\omega}}=s_{X(\omega)}\right\}
\end{aligned}
$$

where $s_{X(\omega)}$ and $s_{\eta}$ are defined as in (3). Let $f_{\omega}$ be the real-valued map defined on $S^{d-1} \times[0,1]$ by $f_{\omega}=s_{X(\omega)}-s_{\eta}$, and define $f_{\omega}^{*}: B(0,1) \rightarrow \mathbb{R}$ as

$$
f_{\omega}^{*}(x)= \begin{cases}\|x\| f_{\omega}\left(\frac{x}{\|x\|},\|x\|\right), & \text { if } x \neq 0 \\ 0, & \text { if } x=0\end{cases}
$$


then, thanks to Bobylev's characterization (Proposition 2),

$$
\begin{aligned}
E & =\{\omega \in \Omega: \operatorname{Ste}(\eta)=0\} \cap\left\{\omega \in \Omega: f_{\omega}^{*} \text { satisfies }(\mathrm{P}-1), \ldots,(\mathrm{P}-6)\right\} \\
& =E_{0} \cap E_{1} \cap \ldots \cap E_{6},
\end{aligned}
$$

where $E_{0}=\{\omega \in \Omega: \operatorname{Ste}(\eta)=0\}$ and $E_{i}=\left\{\omega \in \Omega: f_{\omega}^{*}\right.$ satisfies $\left.(\mathrm{P}-\mathrm{i})\right\}$ for $i=1, \ldots, 6$. Clearly, $E$ is measurable if $E_{0}, \ldots, E_{6}$ are so. Since $\eta$ is a deterministic fuzzy set, $E_{0}$ is either the empty set or the whole $\Omega$; hence $E_{0}$ is measurable. Further, note that each $E_{i}(i=1, \ldots, 4,6)$ can be written as $E_{i}=\left\{\omega: g_{i}(\omega) \leq 0\right\}$ and $E_{5}=\left\{\omega: g_{5}(\omega)<+\infty\right\}$ where

$$
\begin{aligned}
& g_{1}=\sup \left\{\left|\limsup _{y \rightarrow x} f_{\omega}^{*}(y)-f_{\omega}^{*}(x)\right|: x \in B(0,1)\right\}, \\
& g_{2}=\sup \left\{\lambda f_{\omega}^{*}(x)-f_{\omega}^{*}(\lambda x): \lambda \in(0,1], x \in B(0,1)\right\}, \\
& g_{3}=\sup \left\{\|x\| f_{\omega}^{*}\left(\lambda \frac{x}{\|x\|}\right)-\left\|x_{1}\right\| f_{\omega}^{*}\left(\lambda \frac{x_{1}}{\left\|x_{1}\right\|}\right)-\left\|x_{2}\right\| f_{\omega}^{*}\left(\lambda \frac{x_{2}}{\left\|x_{2}\right\|}\right)\right. \\
& \left.\qquad: \lambda \in(0,1], x, x_{1}, x_{2} \in \mathbb{R}^{d} \backslash\{0\}, \text { with } x=x_{1}+x_{2}\right\}, \\
& g_{4}=-\inf \left\{f_{\omega}^{*}(x)+f_{\omega}^{*}(-x): x \in B(0,1)\right\}, \\
& g_{5}=\sup \left\{\frac{\left|f_{\omega}^{*}(x)\right|}{\|x\|}: x \in B(0,1) \backslash\{0\}\right\}, \\
& g_{6}=\left|f_{\omega}^{*}(0)\right| .
\end{aligned}
$$

Clearly $\omega \mapsto g_{i}(\omega)$ are measurable maps and hence $E$ is a measurable event in the $\sigma$-algebra $\mathfrak{F}$.

ITEM (i). Since $\mathbb{I}_{0}$ belongs to $\Theta_{X}, \Theta_{X}$ is not empty.

ITEM (ii). The sufficiency is trivial, let us prove the necessity. Let $E^{c}=\Omega \backslash E=\left\{\omega \in \Omega: \eta \notin \theta_{X(\omega)}\right\}$, by hypothesis $\mathbb{P}(E)=1$ and $\mathbb{P}\left(E^{c}\right)=0$. For every $\omega \in \Omega \cap E$, there exists $Y_{\omega} \in \mathbb{F}$ such that $\eta \oplus Y_{\omega}=X(\omega)$. Let us consider the map

$$
\begin{aligned}
Y: \Omega & \rightarrow \mathbb{F} \\
\omega & \mapsto Y(\omega)= \begin{cases}Y_{\omega}, & \omega \in \Omega \cap E, \\
\mathbb{I}_{0}, & \omega \in E^{c} .\end{cases}
\end{aligned}
$$

Since $s_{Y}=s_{X}-s_{\eta} \mathbb{P}$-almost surely, $s_{Y}$ is measurable. Hence, the map $Y$ defined above, is the FRV we are looking for.

Moreover, let $X \in L^{2}[\Omega ; \mathbb{F}]$, then $s_{X}$ and hence $s_{Y}=s_{X}-s_{\eta}$ belong to $L^{2}[\Omega ; \mathcal{L}]$.

ITEM (iii). Consider $\eta^{1}, \eta^{2} \in \Theta_{X}$. By ITEM (ii) we know that there exist two $\operatorname{FRV} Y^{1}, Y^{2}$ with values in $\mathbb{F}$ such that $\mathbb{P}$-a.s. $\eta^{1} \oplus Y^{1}=X$ and $\eta^{2} \oplus Y^{2}=X$. For any $\lambda \in[0,1]$, the following hold

$$
\lambda\left(\eta^{1} \oplus Y^{1}\right)=\lambda X, \quad(1-\lambda)\left(\eta^{2} \oplus Y^{2}\right)=(1-\lambda) X, \quad \mathbb{P}-\text { a.s. }
$$

so that

$$
\left[\lambda \eta^{1} \oplus(1-\lambda) \eta^{2}\right] \oplus Y=X, \quad \mathbb{P}-\text { a.s. }
$$

with $Y=\lambda Y^{1} \oplus(1-\lambda) Y^{2} \mathbb{P}$-a.s.. Hence $\lambda \eta^{1} \oplus(1-\lambda) \eta^{2} \in \Theta_{X}$.

To prove the last part consider $\eta \in \Theta_{X}$, then $\lambda \eta=\lambda \eta \oplus(1-\lambda) \mathbb{I}_{0} \in \Theta_{X}$ for any $\lambda \in[0,1]$.

ITEM (iv). Consider a sequence $\left\{\eta_{n}\right\}_{n=1}^{\infty} \subset \Theta_{X}$ converging to $\eta \in \mathbb{F}$ in $\left(\mathbb{F}, \delta_{H}^{\infty}\right)$, i.e.

$$
\delta_{H}^{\infty}\left(\eta, \eta_{n}\right) \rightarrow 0, \text { as } n \rightarrow \infty .
$$

We have to prove that $\eta \in \Theta_{X}$.

For any $n \in \mathbb{N}$, let $E_{n}=\left\{\omega \in \Omega: \eta_{n} \in \theta_{X(\omega)}\right\}$ and $Y_{n}$ a FRV as in (ii), then, for any $\omega \in \Omega \cap E_{n}$, $\eta_{n} \oplus Y_{n}(\omega)=X(\omega)$. Further, for any $N \in \mathbb{N}$, there exist $n, m \geq N$ such that, for any $\omega \in \Omega \cap E_{n} \cap E_{m}$,

$$
\delta_{H}^{\infty}\left(Y_{m}(\omega), Y_{n}(\omega)\right)=\delta_{H}^{\infty}\left(\eta_{m}, \eta_{n}\right)<\frac{1}{N}
$$

Thus, the completeness of $\left(\mathbb{F}, \delta_{H}^{\infty}\right)$ guarantees that, for every $\omega \in \Omega \cap \bigcap_{n} E_{n},\left\{Y_{n}(\omega)\right\}_{n \in \mathbb{N}}$ converges w.r.t. $\delta_{H}^{\infty}$ to some $Y_{\omega} \in \mathbb{F}$. Further, for every $\omega \in \Omega \cap \bigcap_{n} E_{n}$ and $n \in \mathbb{N}$ the following inequalities hold

$$
\begin{aligned}
0 \leq \delta_{H}^{\infty}\left(X(\omega), \eta \oplus Y_{\omega}\right) & \leq \delta_{H}^{\infty}\left(X(\omega), \eta_{n} \oplus Y_{n}(\omega)\right)+\delta_{H}^{\infty}\left(\eta_{n} \oplus Y_{n}(\omega), \eta \oplus Y_{\omega}\right) \\
& \leq 0+\delta_{H}^{\infty}\left(\eta_{n}, \eta\right)+\delta_{H}^{\infty}\left(Y_{n}(\omega), Y_{\omega}\right) \rightarrow 0
\end{aligned}
$$


where we use the triangular inequality, the identity $X(\omega)=\eta_{n} \oplus Y_{n}(\omega)$, and the inequality (cf. [4, p.53])

$$
\delta_{H}^{\infty}\left(\nu^{1} \oplus \nu^{2}, \eta^{1} \oplus \eta^{2}\right) \leq \delta_{H}^{\infty}\left(\nu^{1}, \eta^{1}\right)+\delta_{H}^{\infty}\left(\nu^{2}, \eta^{2}\right), \quad \nu^{i}, \eta^{i} \in \mathbb{F}, i=1,2 .
$$

Then $X=\eta \oplus Y \quad \mathbb{P}$-a.s., and $Y$ is the FRV defined by Equation (7). Thus we have the thesis; the limit of the convergent sequence $\left\{\eta_{n}\right\} \subseteq \Theta_{X}$ belongs to $\Theta_{X}$ too.

$\operatorname{ITEM}(\mathbf{v})$. Let us consider a sequence $\left\{\eta_{n}\right\}_{n=1}^{\infty} \subset \Theta_{X}$ converging to $\eta \in \mathbb{F}$ in $\left(\mathbb{F}, d_{2}\right)$, i.e.

$$
d_{2}\left(\eta, \eta_{n}\right) \rightarrow 0, \text { as } n \rightarrow \infty
$$

We have to prove that $\eta \in \Theta_{X}$. In this case, $\left(\mathbb{F}, d_{2}\right)$ is not complete and, hence, we can not repeat exactly all arguments in (iv). In particular, for any $n \in \mathbb{N}$ and for every $\omega \in \Omega \cap E_{n}=\left\{\omega \in \Omega: \eta_{n} \in \theta_{X(\omega)}\right\}$, there exist $Y_{n}(\omega)$ such that $\eta_{n} \oplus Y_{n}(\omega)=X(\omega)$ and, using analogous arguments of those in Proposition 3 ,

$$
d_{2}\left(Y_{m}(\omega), Y_{n}(\omega)\right)=\left(\int_{0}^{1} \int_{S^{d-1}}\left|s_{Y_{m}(\omega)}(\alpha, u)-s_{Y_{n}(\omega)}(\alpha, u)\right|^{2} \mathrm{~d} u \mathrm{~d} \alpha\right)^{\frac{1}{2}}=d_{2}\left(\eta_{m}, \eta_{n}\right) \rightarrow 0,
$$

as $n \rightarrow \infty$ and where $\mathrm{d} \alpha$ and $\mathrm{d} u$ denote the Lebesgue measure on $[0,1]$ and the normalized Lebesgue measure on $S^{d-1}$ respectively. Thus, for every $\omega \in \Omega \cap \bigcap_{n} E_{n},\left\{s_{Y_{n}(\omega)}\right\}_{n \in \mathbb{N}}$ is a Cauchy sequence in the Hilbert space $\mathcal{L}\left(=L^{2}\left[[0,1] \times S^{d-1} ; \mathbb{R}\right]\right)$ and it admits limit in $\mathcal{L}$, namely $f_{\omega}$. Since

$$
\left\|s_{Y_{n}(\omega)}-\left(s_{X(\omega)}-s_{\eta}\right)\right\|_{\mathcal{L}}=\left\|\left(s_{Y_{n}(\omega)}-s_{X(\omega)}\right)+s_{\eta}\right\|_{\mathcal{L}}=\left\|s_{\eta}-s_{\eta_{n}}\right\|_{\mathcal{L}} \rightarrow 0
$$

necessarily we have

$$
s_{Y_{n}(\omega)} \stackrel{L^{2}}{\rightarrow} f_{\omega}=s_{X(\omega)}-s_{\eta}, \quad \forall \omega \in \Omega \cap \bigcap_{n} E_{n} .
$$

Note that, $f_{\omega}$ is not necessarily the support function of some element in $\mathbb{F}$. In other words, for every $\omega \in \Omega \cap \bigcap_{n} E_{n},\left\{Y_{n}(\omega)\right\}_{n \in \mathbb{N}}$ is a Cauchy sequence in the non-complete space $\left(\mathbb{F}, d_{2}\right)$, but under the embedding $j$, Equation (5), we have that the sequence $\left\{j\left(Y_{n}(\omega)\right)\right\}_{n \in \mathbb{N}}=\left\{s_{Y_{n}(\omega)}\right\}_{n \in \mathbb{N}}$ is a Cauchy sequence that admits limit in the Hilbert space $\mathcal{L}$. But, in general, this limit is not the image under $j$ of some element of $\mathbb{F}$. We claim that, for every $\omega \in \Omega \cap \bigcap_{n} E_{n}$, there exists $Y_{\omega} \in \mathbb{F}$ such that $s_{Y_{\omega}}=f_{\omega}=s_{X(\omega)}-s_{\eta}$. This allows us to deduce the thesis because, defining the FRV $Y$ as in Equation (7), we have that $\eta \oplus Y=X$ holds $\mathbb{P}$-a.s..

In fact, let us consider the family $\left\{C_{\alpha}\right\}_{\alpha \in[0,1]}$ of subsets of $\mathbb{R}^{d}$ defined by

$$
C_{\alpha}=\left\{y \in \mathbb{R}^{d}:\langle y, u\rangle \leq f_{\omega}(\alpha, u), \forall u \in S^{d-1}\right\}, \quad \alpha \in[0,1] .
$$

In what follows, let $\omega \in \Omega \cap \bigcap_{n} E_{n}$, we prove that the family $\left\{C_{\alpha}\right\}_{\alpha \in[0,1]}$ satisfies (a), (b), (c) from Lemma 4 , and it defines uniquely a fuzzy set $\nu$ whose support function is, clearly, $f_{\omega}$. Thus the fuzzy set $\nu$ defined in Lemma 4 is just the $Y(\omega)$ in $\mathbb{F}$ we are looking for.

(a). Let $\alpha \in[0,1]$.

$C_{\alpha}$ is non-empty: since $\eta_{\alpha} \subseteq(X(\omega))_{\alpha}$, then for every $u \in S^{d-1}$

$$
f_{\omega}(\alpha, u)=s_{X(\omega)}(\alpha, u)-s_{\eta}(\alpha, u) \geq 0=\langle 0, u\rangle,
$$

i.e. $0 \in C_{\alpha}$.

$C_{\alpha}$ is convex: let $\lambda \in[0,1]$ and $y_{1}, y_{2} \in C_{\alpha}$, for every $u \in S^{d-1}$

$$
\left\langle\lambda y_{1}+(1-\lambda) y_{2}, u\right\rangle \leq \lambda f_{\omega}(\alpha, u)+(1-\lambda) f_{\omega}(\alpha, u)=f_{\omega}(\alpha, u)
$$

i.e. $\lambda y_{1}+(1-\lambda) y_{2} \in C_{\alpha}$.

$C_{\alpha}$ is compact: we have to prove that it is a bounded closed subset of $\mathbb{R}^{d}$. Note that $\{0\} \subseteq \eta_{\alpha} \subseteq(X(\omega))_{\alpha}$, then $s_{X(\omega)}(\alpha, u) \geq s_{\eta}(\alpha, u) \geq 0$ for each $u \in S^{d-1}$ and $s_{X(\omega)}(\alpha, u) \geq s_{X(\omega)}(\alpha, u)-s_{\eta}(\alpha, u)=f_{\omega}(\alpha, u)$. This implies that $\langle y, u\rangle$ is bounded for every $u \in S^{d-1}$ and hence that $C_{\alpha} \subseteq \mathbb{R}^{d}$ is bounded. On the other hand, let $\left\{y_{n}\right\} \subset C_{\alpha}$ be convergent to $y \in \mathbb{R}^{d}$, then, for every $n \in \mathbb{N}$ and $u \in S^{d-1}$,

$$
\left\langle y_{n}, u\right\rangle \leq f_{\omega}(\alpha, u)
$$

and passing to the limit we obtain the same inequality for $y$ and for every $u \in S^{d-1}$; i.e. $y \in C_{\alpha}$. This fact allows us to conclude that $C_{\alpha}$ is closed and hence compact. 
(b). Let $0 \leq \alpha \leq \beta \leq 1$. Note that, for every $n \in \mathbb{N}$ and $u \in S^{d-1}, s_{Y_{n}(\omega)}(\beta, u) \leq s_{Y_{n}(\omega)}(\alpha, u)$. Let $n \rightarrow \infty$, then $f_{\omega}(\beta, u) \leq f_{\omega}(\alpha, u)$ for every $u \in S^{d-1}$; i.e., for every $u \in S^{d-1}$ and $n \in \mathbb{N}, s_{Y_{n}(\omega)}$ and $f_{\omega}$ are non-increasing functions with respect to $\alpha$. Now, let us consider $y \in C_{\beta}$, then for every $u \in S^{d-1}$, $\langle y, u\rangle \leq f_{\omega}(\beta, u) \leq f_{\omega}(\alpha, u)$; i.e. $y \in C_{\alpha}$ and $C_{\beta} \subseteq C_{\alpha}$.

(c). Let $\left\{\alpha_{i}\right\}_{i \in \mathbb{N}} \subset[0,1]$ such that $\alpha_{i} \uparrow \alpha$ as $i$ tends to infinity, that is $\alpha_{i} \leq \alpha_{i+1}$ and $\alpha_{i} \rightarrow \alpha$ as $i \rightarrow \infty$. Because of $\alpha_{i} \leq \alpha$ and (b), we have $C_{\alpha} \subseteq C_{\alpha_{i}}$ and $C_{\alpha} \subseteq \bigcap_{i \in \mathbb{N}} C_{\alpha_{i}}$. It remains to show the opposite inclusion. To do this let $y \in \bigcap_{i \in \mathbb{N}} C_{\alpha_{i}}$, i.e. $y \in C_{\alpha_{i}}$ for all $i \in \mathbb{N}$ or, equivalently,

$$
\langle y, u\rangle \leq f_{\omega}\left(\alpha_{i}, u\right), \quad \text { for every } i \in \mathbb{N}, u \in S^{d-1} .
$$

Note that, for every $u \in S^{d-1}, f_{\omega}(\cdot, u)$ is left-continuous with respect to $\alpha$ because it is the difference of two left-continuous functions (cf. Equation (8)). Hence, for the arbitrariness of $i$ in (9), as $i$ tends to infinity we get $\langle y, u\rangle \leq f_{\omega}(\alpha, u)$; i.e. $y \in C_{\alpha}$.

ITEM (vi). The $D_{\theta}$ case is a consequence of ITEM (v) and of the equivalence of $d_{2}$ and $D_{\theta}$.

The proof in the $D_{\theta}^{\varphi}$ case is exactly the same of ITEM $(\mathrm{v})$ with $\left(\mathcal{L},\langle\cdot, \cdot\rangle_{\theta}^{\varphi}\right)$ and $D_{\theta}^{\varphi}$ substituting $\left(\mathcal{L},\langle\cdot, \cdot\rangle_{\mathcal{L}}\right)$ and $d_{2}$ respectively.

Remark 6 Using the definition of fuzzy sets given in [5], the 0-level of fuzzy sets can be unbounded generalizing our framework. In this case, the space of fuzzy sets is not only separable but also complete since the $D_{\theta}^{\varphi}$ metric isometrically embeds the considered space onto a closed convex cone of a Hilbert space. Under this extended framework, properties of the Hukuhara set (Proposition 5) may be easily obtained. On the other hand, the Steiner point is not always defined and, hence, a characterization point (with similar properties: additivity and continuity) for this extended framework should be found for completeness.

\section{Hukuhara decomposition}

Now, we are ready to prove the decomposition (2) presented in the Introduction that is the main result of this paper. Roughly speaking, (2) allows to decompose uniquely (thanks to a minimization argument) any $X \in L^{2}[\Omega ; \mathbb{F}]$ as the sum of a deterministic convex fuzzy set $H_{X}^{\perp} \in \Theta_{X}$, a FRV $Y$ (that carries the whole "fuzzy randomness" of $X$ ) and a random translation in $\mathbb{R}^{d}$ (that provides the "spatial" location of $X)$.

We present the main result in Corollary 9 as a consequence of the centered case proved in Theorem 7.

Theorem 7 Let $X \in L^{2}[\Omega ; \mathbb{F}]$ with $\mathbf{S t e}(X)=0$. Thus there exists $H_{X}^{\perp} \in \mathbb{F}$ with $\mathbf{S t e}\left(H_{X}^{\perp}\right)=0$ and $Y \in L^{2}[\Omega ; \mathbb{F}]$ with $\mathbf{S t e}(Y)=0 \mathbb{P}$-a.s. such that $X$ decomposes according to

$$
X(\omega)=H_{X}^{\perp} \oplus Y(\omega),
$$

for $\mathbb{P}$-almost all $\omega \in \Omega$. In particular, $H_{X}^{\perp}$ is the unique element in $\mathbb{F}$ that satisfies (10) and minimizes $\Delta_{2}(X, \eta)$ (with $\eta$ varying in $\Theta_{X}$ ); i.e., there exists a unique $H_{X}^{\perp} \in \Theta_{X}$ such that

$$
H_{X}^{\perp}:=\underset{\eta \in \Theta_{X}}{\arg \min } \Delta_{2}(X, \eta)=\underset{\eta \in \Theta_{X}}{\arg \min } \mathbb{E}\left[\left(d_{2}(X, \eta)\right)^{2}\right] .
$$

Hence $Y$ is the unique (except on a $\mathbb{P}$-negligible set) FRV such that its support function is given by $s_{Y}=s_{X}-s_{H_{X}^{\perp}}$. Moreover, $H_{X}^{\perp}$ is a maximal element in $\Theta_{X}$ with respect to the level-wise set inclusion; that is, if $C \in \Theta_{X}$ with $\left(H_{X}^{\perp}\right)_{\alpha} \subseteq C_{\alpha}$ for any $\alpha \in[0,1]$, then $H_{X}^{\perp}=C$.

Corollary 8 Let $X \in L^{2}[\Omega ; \mathbb{F}], D \in \mathbb{F}$ with $\mathbf{S t e}(X)=\mathbf{S t e}(D)=0$ and $X^{\prime}=X \oplus D$. Then $H_{X^{\prime}}^{\perp}=$ $H_{X}^{\perp} \oplus D$.

Corollary 9 Let $X \in L^{2}[\Omega ; \mathbb{F}]$. Thus there exists $H_{X}^{\perp} \in \mathbb{F}$ with $\mathbf{S t e}\left(H_{X}^{\perp}\right)=0$ and $Y \in L^{2}[\Omega ; \mathbb{F}]$ with $\operatorname{Ste}(Y)=0 \mathbb{P}$-a.s. such that $X$ decomposes uniquely according to Equation (2)

$$
X(\omega)=H_{X}^{\perp} \oplus Y(\omega) \oplus \mathbb{I}_{\mathbf{S t e}(X(\omega))},
$$

for $\mathbb{P}$-almost all $\omega \in \Omega$. In particular, it holds

$$
H_{X}^{\perp}=H_{\tilde{X}}^{\perp}, \quad Y=\tilde{Y},
$$


with $H_{\tilde{X}}^{\perp}$ and $\tilde{Y}$ given by Theorem 7 applied to the $F R V \tilde{X}=X \oplus \mathbb{I}_{-} \mathbf{S t e}(X)$.

Moreover, if $D \in \mathbb{F}$ and $X^{\prime}=X \oplus D$ then

$$
X^{\prime}=H_{X^{\prime}}^{\perp} \oplus Y^{\prime} \oplus \mathbb{I}_{\mathbf{S t e}\left(X^{\prime}\right)}=\left(H_{X}^{\perp} \oplus D \oplus \mathbb{I}_{-\operatorname{Ste}(D)}\right) \oplus \tilde{Y} \oplus \mathbb{I}_{\mathbf{S t e}(X)+\operatorname{Ste}(D)}, \quad \mathbb{P}-\text { a.s. }
$$

Proof of Theorem 7. Since $\Theta_{X}$ collects all the elements of $\mathbb{F}$ for which (10) holds, we have to prove that there exists a unique element in $\Theta_{X}$ that minimizes the map $\eta \in \Theta_{X} \rightarrow \mathbb{E}\left[\left(d_{2}(X, \eta)\right)^{2}\right]$.

At first note that $\Theta_{X}$ can be seen as a subset of $L^{2}[\Omega ; \mathbb{F}]$; in fact, for each $\eta \in \Theta_{X}$ the constant map $\omega \mapsto \eta$ is an element of $L^{2}[\Omega ; \mathbb{F}]$ since

$$
\mathbb{E}\left[\left(\sup _{x \in \eta_{0}}\|x\|\right)^{2}\right]=\left(\sup _{x \in \eta_{0}}\|x\|\right)^{2}<+\infty .
$$

Moreover, $\Theta_{X}$ is closed in $\left(L^{2}[\Omega ; \mathbb{F}], \Delta_{2}\right)$ as a consequence of

$$
\Delta_{2}(\nu, \eta)=\left\{\mathbb{E}\left[\left(d_{2}(\nu, \eta)\right)^{2}\right]\right\}^{1 / 2}=d_{2}(\nu, \eta),
$$

for any couples $\nu, \eta \in \mathbb{F}$, and thanks to the fact that $\Theta_{X}$ is closed in $\left(\mathbb{F}, d_{2}\right)$, see Proposition 5 .

Thus the minimization problem is equivalent to prove that there exists a unique projection of $X$ onto $\Theta_{X}$ that is a closed convex subset of $L^{2}[\Omega ; \mathbb{F}]$ endowed with the metric $\Delta_{2}$. Since $\left(L^{2}[\Omega ; \mathbb{F}], \Delta_{2}\right)$ embeds isometrically in the Hilbert space $\left(L^{2}[\Omega ; \mathcal{L}], \mathbb{E}\left[\langle\cdot, \cdot\rangle_{\mathcal{L}}\right]\right)$ through map $J$ (see Section 2), there exists a unique element $H_{X}^{\perp} \in \Theta_{X}$ that realizes the required minimum

$$
H_{X}^{\perp}=\underset{\eta \in \Theta_{X}}{\arg \min } \mathbb{E}\left[\left(d_{2}(X, \eta)\right)^{2}\right] .
$$

As a consequence of $H_{X}^{\perp} \in \Theta_{X}$ and of (ii) in Proposition 5, the FRV $Y$ is defined through its support function $s_{Y}=s_{X}-s_{H_{X}}$.

Finally, let $C$ be as in the thesis; thus inclusions $\left(H_{X}^{\perp}\right)_{\alpha} \subseteq C_{\alpha} \subseteq X_{\alpha}$ imply $s_{X}-s_{C} \leq s_{X}-s_{H_{X}}$. Then, by definitions of $H_{X}^{\perp}$ and $d_{2}$, necessarily $C=H_{X}^{\perp}$ holds.

The chosen notation wants to recall the line of the proof; $H_{X}^{\perp}$ is obtained as the (necessarily unique) projection of the FRV $X$ on its Hukuhara set $\Theta_{X}$. Further, we want to stress out that the suffix $X$ does not mean that $H_{X}^{\perp}$ is random; in fact, it does not depend on $\omega$ but rather it is a deterministic element of $\mathbb{F}$ (that is a constant element in $L^{2}[\Omega ; \mathbb{F}]$ ) that depends on the whole map $\omega \mapsto X(\omega)$. A qualitative graphical interpretation of Theorem 7 is given in Figure 1.

About different metrics and open problems: Although in this paper different metric for fuzzy sets are used, the decomposition in Theorem 7 is just proved for the $d_{2}$ metric. Thus, the following question arises spontaneously: what can we say if $d_{2}$ is replaced with another metric? Note that, two facts are mainly involved in the proof of Theorem 7 :

1. $\Theta_{X}$ is a non-empty, closed convex subset of $L^{2}[\mathbb{F}]$ with respect to $d_{2}$.

2. $\left(L^{2}[\mathbb{F}], d_{2}\right)$ embeds isometrically in a Hilbert space.

These facts allow to project $X$ onto $\Theta_{X}$ in a unique way. As a consequence, arguments in Theorem 7 still hold for every metric that satisfies the preceding properties. This is the case of $D_{\theta}$ but not of $\delta_{H}^{\infty}$ (because a Hilbert space is not available). Anyway, changing metric several questions may arise.

Let us consider at first the case of $D_{\theta}$. Although $d_{2}$ and $D_{\theta}$ induce the same topology on $\mathbb{F}$, the projection $\widetilde{H_{X}^{\perp}}$ obtained w.r.t. $D_{\theta}$, in general, differs from $H_{X}^{\perp}$. It is a conjecture of the authors that $\widetilde{H_{X}^{\perp}}=H_{X}^{\perp}$. In the case of $\delta_{H}^{\infty}, L^{2}[\mathbb{F}]$ can not be embedded in a Hilbert space and, hence, a projection theorem can not be used. Anyway, the map $\delta_{H}^{\infty}(X, \cdot)$ is continuous and convex and, hence, admits at least a minimum on $\Theta_{X}$. Nevertheless, the minimum is not necessarily achieved in a unique way and, even if it was unique, it should be compared with $H_{X}^{\perp}$ and $\widetilde{H_{X}^{\perp}}$. Author's conjecture is that both $\widetilde{H_{X}^{\perp}}$ and $H_{X}^{\perp}$ minimize $\mathbb{E}\left[\delta_{H}^{\infty}(X, \cdot)^{2}\right]$ on $\Theta_{X}$. 


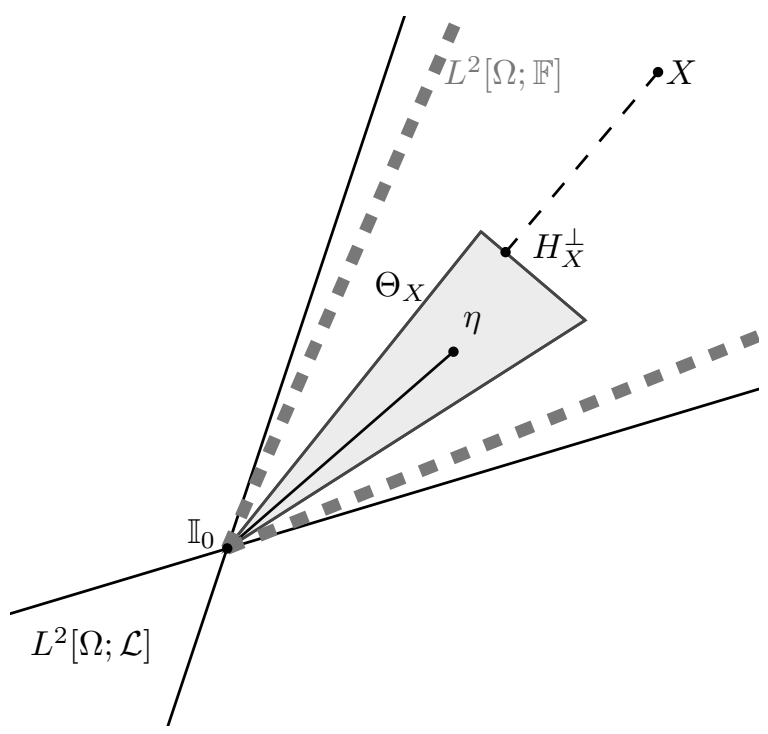

Figure 1: A qualitative graphical interpretation of some results of Section 3 and Section 4. In particular, $\Theta_{X}$ is represented as a closed convex subset of $\mathbb{F}$ containing the origin and such that, for any $\eta \in \Theta_{X}$ and $\lambda \in[0,1], \lambda \eta \in \Theta_{X}$. Hence, $H_{X}^{\perp}$ is the projection of $X$ on $\Theta_{X}$, as a subset of $L^{2}[\Omega ; \mathbb{F}]$, with respect to the metric $\mathbb{E}\left[d_{2}(\cdot, \cdot)^{2}\right]$, this also guarantees the uniqueness of $H_{X}^{\perp}$ since the cone $L^{2}[\Omega ; \mathbb{F}]$ is embeddable in the Hilbert space $L^{2}[\Omega ; \mathcal{L}]$ through the isometry $X \mapsto j(X)$. Finally the following inclusions or embeddings are qualitatively represented: $\Theta_{X} \subseteq \mathbb{F} \hookrightarrow L^{2}[\Omega ; \mathbb{F}] \hookrightarrow L^{2}[\Omega ; \mathcal{L}]$.

Fuzzy random translations. Theorem 7 allows us to characterize fuzzy random translation by means of the Aumann expectation.

Definition 10 A FRV $X$ is a translation if there exists $\nu \in \mathbb{F}$ with $\operatorname{Ste}(\nu)=0$ such that

$$
X(\omega)=\nu \oplus \mathbb{I}_{\text {Ste }(X)} .
$$

Clearly, every Gaussian FRV $X$ is a FRV translation with $\nu \oplus \mathbb{I}_{\mathbb{E}[\operatorname{Ste}(X)]}=\mathbb{E} X$. Another sufficient condition for $X$ to be a FRV translation is given by Proposition 11, while a necessary and sufficient condition is stated in Proposition 12.

Proposition 11 Let $X$ be a FRV such that $\mathbb{E} X=\mathbb{I}_{c}$ where $c \in \mathbb{R}^{d}$. Then $X=\mathbb{I}_{\xi} \mathbb{P}-$ a.s. for some random element $\xi$ in $\mathbb{R}^{d}$. (Clearly $X$ is a FRV translation.)

Proof. Thesis may be obtained as in [2, Theorem 6], or, whenever $X \in L^{2}[\Omega ; \mathbb{F}]$, as corollary of the Theorem 7 and Proposition 12.

Let $X$ be a FRV translation, and $\widetilde{X}=X \oplus \mathbb{I}_{-\mathbf{S t e}(X)}$. Then it easy to check that

$$
X=H_{\tilde{X}}^{\perp} \oplus \mathbb{I}_{\text {Ste }(X)}, \quad \mathbb{P}-\text { a.s. }
$$

Proposition 12 Let $X \in L^{2}[\Omega ; \mathbb{F}] . X$ is a FRV translation if and only if $H_{\widetilde{X}}^{\perp}$ satisfies

$$
\mathbb{E} X=H_{\tilde{X}}^{\perp} \oplus \mathbb{I}_{\mathbb{E}[\operatorname{Ste}(X)]}
$$

with $\mathbb{E} X$ being the Aumann expectation; in other words, $H_{\widetilde{X}}^{\perp}$ is equal to $\mathbb{E} X$ up to a translation.

Proof. For the "only if" part, in order to obtain Equation (12), it is sufficient to compute the expectation in Equation (11).

"If" part. For the sake of simplicity, let us assume that $\operatorname{Ste}(X)=0$, a straightforward argument extends the result in the more general case of a FRV with non-null Ste $(X)$. Then, in term of support functions, Equation (10) becomes

$$
s_{X}=s_{H_{X}^{\perp}}+s_{Y}=s_{\mathbb{E} X}+s_{Y}, \quad \mathbb{P}-\text { a.s. }
$$


where we use the fact that $H_{X}^{\perp}=\mathbb{E} X$. Computing expectation of both sides and using (6), we get $s_{\mathbb{E} Y}=0$. Hence $Y=\mathbb{I}_{\xi}$ a.s. for some random element $\xi$ in $\mathbb{R}^{d}$ (cf. [2]).

Remark 13 Whenever $X \in L^{2}[\Omega ; \mathbb{F}]$, in view of Theorem 7 and Proposition 12, we get a proof of Proposition 11. In fact, suppose that $\mathbb{E} X=\mathbb{I}_{c}$ for some $c \in \mathbb{R}^{d}$, and compute expectation of both sides in Equation (10)

$$
\mathbb{I}_{c}=\mathbb{E} X=H_{X}^{\perp} \oplus \mathbb{E} Y \text {. }
$$

Hence, for any $\alpha \in[0,1],\left(H_{X}^{\perp}\right)_{\alpha}$ is a subset of $\{c\}$ up to a translation, that is $\left(H_{X}^{\perp}\right)_{\alpha}$ is a singleton as well as $(\mathbb{E} Y)_{\alpha}$. Then $H_{X}^{\perp}=\mathbb{I}_{c^{\prime}}$ for some $c^{\prime} \in \mathbb{R}^{d}$, i.e. $H_{X}^{\perp}$ equals $\mathbb{E} X$ up to a translation and, by Proposition $12, X$ is a FRV translation that implies $Y=\mathbb{I}_{\xi}$ for some random element in $\mathbb{R}^{d}$. Finally, Equation (10) becomes

$$
X=H_{X}^{\perp} \oplus Y=\mathbb{I}_{c^{\prime}} \oplus \mathbb{I}_{\xi}=\mathbb{I}_{\xi^{\prime}},
$$

that is the thesis of Proposition 11.

A decomposition example. Remark 14 provides an example of an $X$ in $L^{2}[\Omega ; \mathbb{F}]$ with $\mathbf{S t e}(X)=0$ for which $\mathbb{E}[X] \neq H_{X}^{\perp}$ and for which $H_{X}^{\perp}$ is not necessarily $\mathbb{I}_{0}$; i.e., in terms of Proposition $12, X$ is not a translation but its deterministic part $H_{X}^{\perp}$ in the decomposition (10) is not just reduced to the origin.

Remark 14 Let $\mathbb{R}^{d}=\mathbb{R},\left(\Omega=[0,1], \mathcal{B}_{[0,1]}, \mathbb{P}\right)$ where $\mathcal{B}_{[0,1]}$ denotes the Borel $\sigma$-algebra on $[0,1]$ w.r.t. the euclidean metric and $\mathbb{P}=\mu$ is the Lebesgue measure. Let $X$ be the FRV defined by $X:=\mathbb{I}_{[-\omega, \omega]}$, for any $\omega \in[0,1]$ (actually $X$ is a random sets, but similar arguments can be repeated either for the triangular FRV $Y=(-\omega, 0, \omega)$ or for the trapezoidal FRV $X \oplus Y=(-2 \omega,-\omega, \omega, 2 \omega))$. Clearly $X \in L^{2}[\Omega ; \mathbb{F}]$ and $\operatorname{Ste}(X)=0$. Moreover,

$$
f_{m}(\omega):=\min X_{1}(\omega)=-\omega \quad \text { and } \quad f_{M}(\omega):=\max X_{1}(\omega)=\omega
$$

are integrable selections of the 1-level $X_{1}$. Obviously, any other integrable selection $f$ of $X_{1}$ satisfies

$$
f_{m}(\omega) \leq f(\omega) \leq f_{M}(\omega), \quad \text { for each } \omega \in[0,1]
$$

Then

$$
-\frac{1}{2}=\mathbb{E} f_{m} \leq \mathbb{E} f \leq \mathbb{E} f_{M}=\frac{1}{2}
$$

and, by the convexity of Aumann expectation and because $X_{1}=X_{\alpha}$ for any $\alpha \in[0,1], \mathbb{E} X_{1}=\left[-\frac{1}{2}, \frac{1}{2}\right]=$ $\mathbb{E} X_{\alpha}$, that is $\mathbb{E} X=\mathbb{I}_{\left[-\frac{1}{2}, \frac{1}{2}\right]}$.

We prove that $\mathbb{E} X \notin \Theta_{X}$ and hence, by Proposition $12, X$ is not a FRV translation. In fact, note that

$$
X \ominus_{H} \mathbb{E} X=\mathbb{I}_{[-\omega, \omega]} \ominus_{H} \mathbb{I}_{\left[-\frac{1}{2}, \frac{1}{2}\right]}= \begin{cases}\mathbb{I}_{\left[-\omega+\frac{1}{2}, \omega-\frac{1}{2}\right]}, & \omega>\frac{1}{2}, \\ \mathbb{I}_{0}, & \omega=\frac{1}{2}, \\ \text { it does not exist, } & \omega<\frac{1}{2},\end{cases}
$$

implies

and hence $\mathbb{E} X \notin \Theta_{X}$.

$$
\mathbb{P}\left(\mathbb{E} X \in \theta_{X}\right)=\mathbb{P}\left(\text { there exists } X \ominus_{H} \mathbb{E} X\right)=\mathbb{P}\left(\omega \geq \frac{1}{2}\right)=\frac{1}{2},
$$

Actually we can show that $\Theta_{X}=\left\{\mathbb{I}_{0}\right\}$ and hence $H_{X}^{\perp}=\mathbb{I}_{0}$. In fact, by absurd let $\eta \in \Theta_{X}$ with $\eta \neq \mathbb{I}_{0}$, then there exists $\alpha \in[0,1]$ such that $\eta_{\alpha}=[a, b]$ with $a<b$ and there exists $X_{\alpha} \ominus_{H} \eta_{\alpha}$, here $\ominus_{H}$ is considered as the Hukuhara difference for subsets in $\mathbb{R}$. On the other hand

$$
[-\omega, \omega] \ominus_{H}[a, b]= \begin{cases}{[-\omega-a, \omega-b],} & \omega-b>-\omega-a \\ \left\{-\frac{b+a}{2}\right\}, & \omega=\frac{b-a}{2}, \\ \text { it does not exist, } & \omega<\frac{b-a}{2},\end{cases}
$$

and, as consequence,

$$
\mathbb{P}\left([-\omega, \omega] \ominus_{H}[a, b] \text { does not exist }\right)=\mu\left[\left(-\infty, \frac{b-a}{2}\right) \cap[0,1]\right]=\frac{b-a}{2}>0
$$


(recall that, by hypothesis, $b>a$ ). This is an absurd since $\eta \in \Theta_{X}$ by hypothesis. Thus $\Theta_{X}=\left\{\mathbb{I}_{0}\right\} \neq$ $\mathbb{E} X=\mathbb{I}_{\left[-\frac{1}{2}, \frac{1}{2}\right]}$.

Finally, in order to introduce a more general example, let us consider

$$
X=\mathbb{I}_{[-\omega, \omega]} \oplus \mathbb{I}_{\left[-\frac{1}{2}, \frac{1}{2}\right]}=\mathbb{I}_{\left[-\omega-\frac{1}{2}, \omega+\frac{1}{2}\right]}
$$

so that, from Corollary 8 , we immediately obtain that

$$
\mathbb{I}_{[-1,1]}=\mathbb{E} X \neq H_{X}^{\perp}=\mathbb{I}_{\left[-\frac{1}{2}, \frac{1}{2}\right]}
$$

Note that, this is a case in which $H_{X}^{\perp}$ is different from $\mathbb{I}_{0}$.

\section{Conclusion}

In this paper, we have proven that any square integrable FRV can be decomposed as $X=H_{X}^{\perp} \oplus Y \oplus \mathbb{I}_{\text {Ste }(X)}$, where $H_{X}^{\perp}$ is a unique deterministic fuzzy convex compact set (i.e. in $\mathbb{F}$ ) and $Y$ is an element of $L^{2}[\Omega ; \mathbb{F}]$ with $\operatorname{Ste}(Y)=0$. The method used to obtain $H_{X}^{\perp}$ is a particular case where the problem posed in $[7$, p.174-175] is well-solved by defining the Hukuhara set $\Theta_{X}$. In this view, $H_{X}^{\perp}$ may be interpreted as an expectation for $X$ that satisfies some of the properties, listed in [7, p.190] for random closed sets but trivially extendible in the fuzzy case, of a "reasonable" expectation of $X$.

The decomposition theorem proposed in Section 4 could not be compared with the fuzzy regression problem stated in [13]. In fact, in that paper, the authors look for the best linear approximation function of a given square integrable FRV $Y$ by another square integrable FRV $X$, studying the minimization problem

$$
\inf _{a \in \mathbb{R}, \eta \in \mathbb{F}} \mathbb{E}\left[d_{2}(Y, a X \oplus \eta)^{2}\right] .
$$

Besides the open problems exposed in Section 4 (concerning the use of different metrics), future works may consider the possibility to relax some hypothesis. As example, replacing $\mathbb{R}^{d}$ with an Hilbert or a Banach space (problems may arise considering the embedding $j$ and hence the closure of the Hukuhara set $\Theta_{X}$ ), or dropping convexity hypothesis and hence stating a decomposition theorem for a fuzzy random element whose level sets are not necessarily convex. Finally, note that we restricted our studies to the existence of a such $H_{X}^{\perp}$. However, it is certainly interesting to establish whenever $H_{X}^{\perp}$ could be explicitly computed or approximated, though even in particular cases.

\section{Acknowledgements}

The authors would like to thank Prof. V. Capasso for the helpful discussions during the preparation of this paper. The authors are also very grateful to the anonymous referees for the valuable comments that helped to improve the paper, especially for suggesting the use of the $D_{\theta}^{\varphi}$ metric and for having pointed out the possibility of an extension to the unbounded support case.

\section{References}

[1] V. N. Bobylev. Support function of a fuzzy set and its characteristic properties. Mathematical Notes, 37, 281-285, 1985.

[2] E. G. Bongiorno. A Note on Fuzzy Set-Valued Brownian Motion. Statistics and Probability Letters, $82,827-832,2012$.

[3] A. Colubi, J. S. Dominguez-Menchero, M. Lopez-Diaz, D. A. Ralescu. A $D_{E}[0,1]$ representation of random upper semicontinuous functions. Proceedings of the American Mathematical Society, 130, 3237-3242, 2002.

[4] P. Diamond, P. Kloeden. Metric spaces of fuzzy sets: theory and applications. Wiley, 1994.

[5] G. González-Rodríguez, A. Colubi, M. A. Gil. Fuzzy data treated as functional data: A one-way ANOVA test approach. Computational Statistics and Data Analysis, 56, 943-955, 2012. 
[6] S. Li, Y. Ogura, V. Kreinovich. Limit Theorems and Applications of Set-Valued and Fuzzy SetValued Random Variables. Kluwer Academic Publishers Group, Dordrecht, 2002.

[7] I. Molchanov. Theory of random sets. Springer. (2005)

[8] W. Näther. Linear Statistical Inference for Random Fuzzy Data. Statistics, 29:3, 221-240, 1997.

[9] W. Näther. On random fuzzy variables of second order and their application to linear statistical inference with fuzzy data. Metrika, 51, 201-221, 2000.

[10] A. B. Ramos-Guajardo, A. Colubi, G. González-Rodríguez, M. A. Gil. One-sample tests for a generalized Fréchet variance of a fuzzy random variable. Metrika, 71, 185-202, 2010.

[11] W. Trutschnig, G. González-Rodríguez, A. Colubi, M. A. Gil. A new family of metrics for compact, convex (fuzzy) sets based on a generalized concept of mid and spread. Information Sciences, 179, 3964-3972, 2009.

[12] T. Vetterlein, M. Navara. Defuzzification using Steiner points. Fuzzy Sets and Systems, 157, 14551462, 2006.

[13] A. Wünsche, W. Näther. Least-squares fuzzy regression with fuzzy random variables. Fuzzy Sets and Systems, 130, 43-50, 2002. 